\title{
BCL10 : rôle ambigu dans la cascade apoptotique et la tumorigenèse
}

Dans les tumeurs lymphoïdes, la présence de translocations récurrentes impliquant les gènes codant pour les récepteurs de l'antigène a déjà permis l'identification des gènes partenaires, tels $c-m y c$ ou $b c l 2$, jouant un rôle crucial dans la physiologie et la pathologie cellulaires. Même si le chemin parcouru entre l'identification du gène cible et l'élucidation des mécanismes l'impliquant dans la tumorigenèse est parfois long, cette stratégie reste d'actualité.

Deux collaborations internationales distinctes, rassemblant chacune plusieurs équipes dans différents laboratoires, ont ainsi récemment caractérisé, dans certains lymphomes dérivés du tissu lymphoïde associé aux muqueuses (ou lymphomes de MALT, mucosa associated lymphoid tissue) $\left(\mathrm{m} / \mathrm{s}\right.$ 1993, $n^{\circ} 12, \mathrm{p} .1422$ et p. 1423), un gène, normalement situé sur le chromosome 1 en position 1p22, qui, dans cette maladie, se trouve à proximité des gènes codant pour la chaîne lourde des immunoglobulines (Ig) sur le chromosome 14, en position 14q32 [1, 2]. Le segment génomique du chromosome 1 impliqué dans la translocation a été cloné en utilisant une stratégie de PCR inverse [3]. Un transcrit complet de 2,8 kb désigné $b c l 10$, a été déduit de la séquence de ce fragment génomique. Il est faiblement exprimé dans plusieurs tissus normaux. Le gène bcl10 contient 4 exons occupant environ $11,7 \mathrm{~kb}$ sur la bande chromosomique 1p22. Dans les différents cas de lymphomes de MALT analysés, l'intégrité du fragment génomique impliqué dans la translocation $t(1 ; 14)(p 22 ; q 32)$ qui juxtapose $b c l 10$ en 5 ' des segments $\mathrm{J}^{\mathrm{H}}$ des gènes d'Ig sur le chromosome 14 dérivatif est conservée. Il en résulte une surexpression du transcrit de $2,8 \mathrm{~kb}$ de bcl10 dans les cellules porteuses de la translocation.

Dans les tissus normaux, bcl10 code pour une protéine de 233 acides aminés, d'un poids moléculaire estimé à $26 \mathrm{kDa}$. Cette protéine a de fortes homologies avec la protéine E10 du virus équin EHV-2 du groupe des herpès virus. L'extrémité amino-terminale recèle une région de 90 acides aminés de type CARD (caspase recruitment domain), qui correspond à un domaine impliqué dans l'oligomérisation et le recrutement des caspases [4]. Simultanément à sa caractérisation dans la translocation $\mathrm{t}(1 ; 14)$, la protéine cellulaire BCL10 était aussi identifiée par plusieurs autres équipes en raison de son homologie avec la séquence CARD de la protéine virale E10 [5-9]. Ces observations nous en apprenaient un peu plus sur la fonction de BCL10. La surexpression de $b c l 10$ dans divers types cellulaires entraîne une augmentation de l'apoptose, qu'elle survienne spontanément ou en réponse à l'activation de Fas ou du récepteur du TNF de type I (TNF-RI). Divers inhibiteurs de la cascade apoptotique, CrmA, p35 et les protéines cIAP ainsi que le peptide z-VAD-fmk, inhibaient l'apoptose induite par BCL10, de même qu'un mutant négatif de la caspase-9. De plus, comme le font d'autres molécules de la machinerie apoptotique, BCL10 est capable de stimuler l'activité de NF-кB. Des expériences de cotransfections ont indiqué que bcl10 intervenait en amont de la kinase acti-

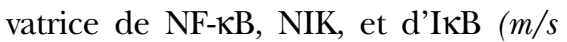
1999, $n^{\circ} 3$, p. 419 et $n^{\circ} 5$, p. 748). L'intégrité du domaine CARD de BCL10 est indispensable à ses deux fonctions, activation de NF-אB, et contrôle de la cascade apoptotique. Même si les données de l'un des groupes ayant identifié l'homologue cellulaire de E10 indiquaient que l'activité de cette protéine était moindre que celle de la protéine virale [5], l'ensemble de ces résultats montre que BCL10 est un nouvel intervenant dans la machinerie apoptotique.

Dès lors, le rôle de BCL10 dans la lymphomagenèse méritait d'être précisé. L'étude des ADN complémentaires (ADNc) issus des tissus tumoraux a révelé un grand nombre d'altérations de la séquence codante de $b c l 10$, qu'il s'agisse de substitutions de bases, de délétions plus ou moins importantes certaines touchant 16 à 33 nucléotides, d'autres 1 ou 2, ou d'insertions de 1 à 2 nucléotides [1, 2]. Certaines de ces modifications altéraient la séquence codante mais la plupart modifiaient le cadre de lecture ce qui résultait en une protéine $\mathrm{BCl10}$ tronquée, parfois dans son domaine CARD, parfois à l'extrémité carboxyterminale. Des expériences de transfection montraient que les clones porteurs d'altérations du domaine CARD avaient perdu la capacité d'activer l'apoptose et NF- $\kappa B$, tandis que ceux tronqués à l'extrémité carboxy-terminale, s'ils étaient capables d'activer NF-KB, avaient perdu leur fonction pro-apoptotique (figure 1). On pouvait dès lors concevoir que ces modifications de BCL10, par l'altération des mécanismes effecteurs d'apoptose et la persistance de l'activation de NF-kB qu'elles entraînaient, conféraient aux cellules où elles étaient présentes un avantage de croissance ou de survie. L'observation, dans des expériences de co-transfection dans des fibroblastes primaires de certains mutants de $b c l 10$ avec ras et un mutant de p53 (ou de E1a), d'une augmentation du nombre de transformants, tandis que bcl10 sauvage diminuait ce nombre, confortait cette hypothèse [1].

Cependant, la présence d'ADNc altérés de bcl10 dans de nombreuses autres tumeurs lymphoïdes ou solides (mésothéliome, tératocarcinome)* révélée par l'équipe de $M$. Dyer jetait un trouble [1]. De nombreuses équipes cherchèrent alors à préciser ces résultats en étudiant la structure génomique de bcl10 dans diverses tumeurs [10-14]. En fait, les altérations somatiques de bcl10 dans les tumeurs sont très rares (même si les techniques de SSCP utilisées ne mettent théoriquement pas à l'abri de résultats faussement négatifs lorsque les cellules porteuses de l'anomalie recherchée sont diluées dans l'échantillon étu-

\footnotetext{
* Ces altérations sont repertoriées sur un site consacré à bcl10 (http://www.icr.ac.uk/haemcyto/bcldata/index.htm).
} 


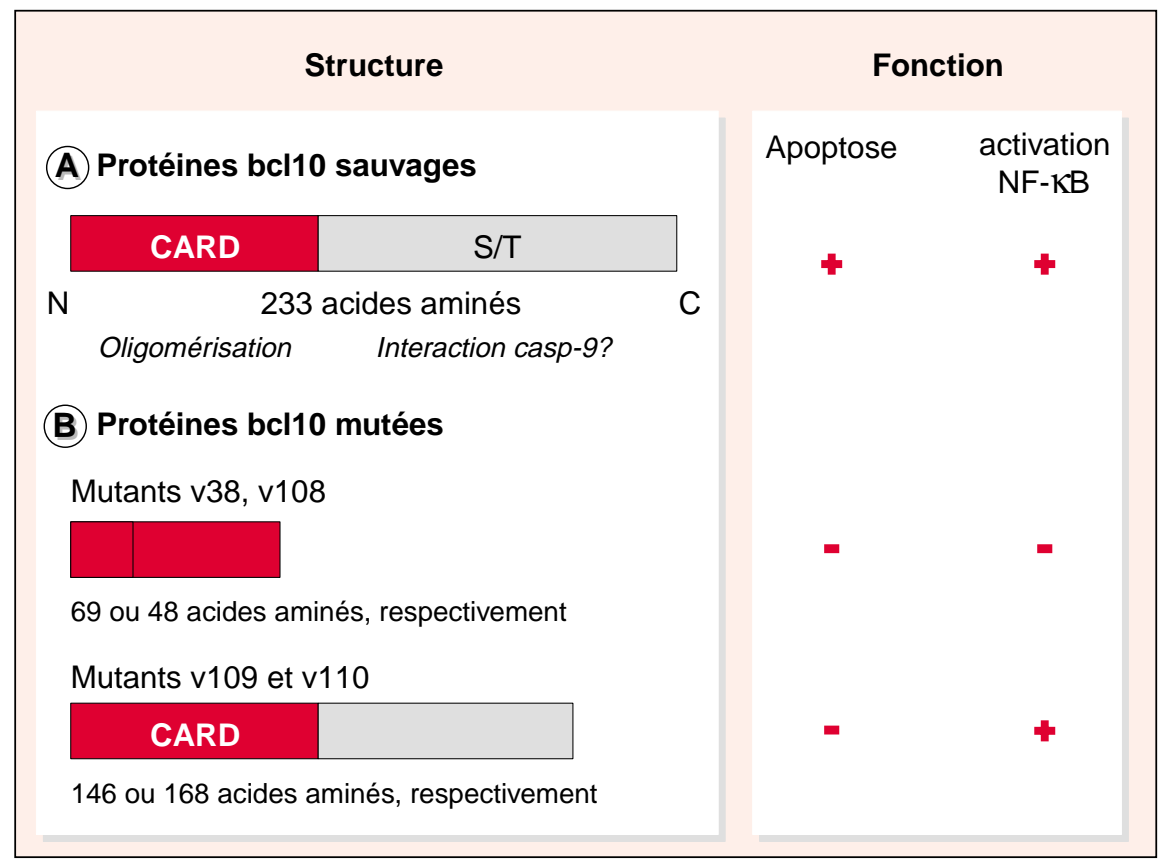

Figure 1. Structure de bcl10 sauvage et de ses mutants. La protéine codée par bcl10 sauvage comprend un domaine de type CARD caractéristique des caspases d'environ 90 acides aminés, qui est indispensable à son oligomérisation et à son activité pro-apoptotique. $S / T$ désigne une région riche en sérines et en thréonines qui est le siège probable de phosphorylation. L'interaction de bc/10 avec la caspase-9 pourrait se faire par l'extrémité carboxy-terminale [6]. Les mutants [2] v38 et v108 (respectivement 68 et 48 acides aminés) sont tronqués et mutés dans le domaine CARD et n'ont plus ni d'activité pro-apoptotique ni d'interactions avec la voie NF-KB. Les mutants v109 et v110 sont tronqués dans le domaine carboxy-terminal et ont gardé la capacité d'activer NF-KB mais ont perdu leur activité pro-apoptotique.

dié), et les nombreux ADNc mutés détectés dans les cellules ne sont pas expliqués par la seule transcription du gène altéré. $\mathrm{Si}$ on regarde de plus près les altérations des $\mathrm{ADNc}$ initialement décrites pour comprendre cette apparente contradiction, on constate que certaines correspondent à des anomalies d'épissage entre l'exon 3 et l'exon 4 (notamment les délétions de $16 \mathrm{pb}$ et $33 \mathrm{pb}$ ) résultant probablement de l'existence de trois sites d'épissage distincts, tandis que d'autres correspondent vraisemblablement à de simples polymorphismes. Il persiste cependant des clones d'ADNc porteurs de mutations isolées ou de délétions/insertions, survenant notamment dans des brins de séquences mononucléotidiques (polyA ou polyT) et qui ne semblent pas retrouvées dans l'ADN somatique des tumeurs étudiées. Certaines de ces modifications paraissaient isolées, d'autres étaient retrou- obtenus à partir d'un même tissu suggérant l'accumulation d'événements au cours du processus tumoral. L'identification de la séquence de certains mutants dans trois laboratoires distincts rend par ailleurs très improbable la possibilité d'un artifice de PCR, de clonage, ou de séquençage. Ces altérations de BCL10 surviendraient donc dans certains cas à une étape post-transcriptionnelle et les mécanismes moléculaires à leur origine restent hypothétiques. Les mécanismes d'édition des ARN messagers connus chez les plantes ont parfois aussi été décrits pour certaines protéines eucaryotes $[15,16]$. La place éventuelle de tels mécanismes dans les altérations de BCL10 qui sont observées mérite cependant d'être précisée, d'autant que des $\mathrm{ADNc}$ de $b c l 10$ altérés ont été clonés dans des cellules lymphoïdes non transformées. Enfin, des résultats préliminaires récents (voir discussion des références $[11,12]$ dans le même numéro de Cell) indiquent que des mutations somatiques du gène bcl10 existeraient dans certains cas de lymphome de MALT.

Quelles sont les conclusions - provisoires - de ces premiers résultats? Un gène codant pour une molécule de la cascade apoptotique se trouve dans la région ciblée par une translocation chromosomique présente dans les lymphomes B, et des altérations de la structure et de l'expression de ce gène pourraient participer aux mécanismes de la transformation. Il s'agit là - à côté de l'exemple initial de bcl2d'un nouveau modèle de tumorigenèse impliquant un défaut d'apoptose, qui doit aussi être rapporté à l'observation de rares mutations des gènes fas ou caspase- 8 dans certains lymphomes [17]. Les mécanismes à l'origine des altérations des séquences codantes de $b c l 10$ dans les tissus tumoraux restent obscurs et le rôle des séquences tronquées dans la transformation cellulaire devra être montré. En particulier, il est important d'identifier, dans les tissus tumoraux, la présence et l'activité des protéines ainsi produites. Il sera aussi intéressant de déterminer la place des anomalies de BCL10 dans la genèse des lymphomes de MALT, qui représentent déjà un modèle de cancérogenèse particulier par l'implication d'une infection par Helicobacter pylori dans les étapes initiales de ces tumeurs. La place de la translocation $\mathrm{t}(1 ; 14)$ - relativement rare dans les lymphomes de MALT dans les anomalies de structure et d'expression de bcl10 devra aussi être précisée. La juxtaposition de bcl10 à proximité des gènes d'Ig est-elle toujours associée à une dérégulation de son expression, un mécanisme déjà connu, notamment pour c-myc? Bcl10 constitue-t-il un gène "cible» pour des mutations somatiques rappelant celles observées dans les gènes d'Ig dans les cellules B, un mécanisme déjà évoqué pour l'activation du gène laz3/bcl6 ? Toutes ces questions permettront de mieux appréhender le rôle de bcl10 dans les tumeurs tandis que l'étude de son oligomérisation, de son mode d'interaction avec la pro-caspase-9 [6] et de sa phosphorylation possible à l'extrémité carboxyterminale préciseront la place de BCL10 dans la cascade apoptotique. 
1. Willis TG, Jadayel DM, Du MQ et al. Bcl10 is involved in $\mathrm{t}(1 ; 14)$ (p22; 932$)$ of MALT B cell lymphoma and mutated in multiple tumor types. Cell $1999 ; 96: 35-45$

2. Zhang Q, Siebert R, Yan M, et al. Inactivating mutations and overexpression of BCL10, a caspase recruitment domain-containing gene, in MALT lymphoma with $\mathrm{t}(1 ; 14)(\mathrm{p} 22 ; \mathrm{q} 32)$. Nat Genet 1999; 22 : 63-8.

3. Willis TG, Jadayel DM, Coignet LJ, et al. Rapid molecular cloning of rearrangements of the IGHJ locus using long-distance inverse polymerase chain reaction. Blood 1997; 90 : 2456-64.

4. Chou JJ, Matsuo H, Duan H, Wagner G. Solution structure of the RAIDD CARD and model for CARD/CARD interaction in caspase-2 and caspase-9 recruitment. Cell 1998; 94: 171-80.

5. Thome M, Martinon F, Hofmann K, et al. Equine herpesvirus-2 E10 gene product, but not its cellular homologue, activates NF-kappaB transcription factor and c-Jun N-terminal kinase. J Biol Chem 1999; 274: 9962-8.

6. Yan M, Lee J, Schilbach S, Goddard A, Dixit V. mE10, a novel caspase recruitment domaincontaining proapoptotic molecule. J Biol Chem $1999 ; 274: 10287-92$

7. Srinivasula SM, Ahmad M, Lin JH, et al. CLAP, a novel caspase recruitment domain-containing protein in the tumor necrosis factor receptor pathway, regulates NF-kappaB activation and apoptosis. J Biol Chem 1999; 274: 17946-54.

8. Koseki T, Inohara N, Chen S, et al. CIPER, a novel NF kappaB-activating protein containing a caspase recruitment domain with homology to Herpesvirus2 protein E10. J Biol Chem 1999; 274: 9955-61.

9. Costanzo A, Guiet C, Vito P. c-E10 is a caspaserecruiting domain-containing protein that interacts with components of death receptors signaling pathway and activates nuclear factor-kappaB. $J$ Biol Chem 1999; 274: 20127-32.

10. Lambers AR, Gumbs C, Ali S, et al. Bcl10 is not a target for frequent mutation in human carcinomas. Br J Cancer 1999; 80 : 1575-6.

11. Apostolou S, De Rienzo A, Murthy SS, Jhanwar SC, Testa JR. Absence of BCL10 mutations in human malignant mesothelioma. Cell 1999; 97 : $684-8$.

12. Fakruddin JM, Chaganti RS, Murty VV. Lack of BCL10 mutations in germ cell tumors and B cell lymphomas. Cell 1999; 97: 683-8.

13. Stone JG, Rowan AJ, Tomlinson IP, Houlston RS. Mutations in Bcl10 are very rare in colorectal cancer. Br J Cancer 1999; 80 : 1569-70.

14. van Schothorst EM, Mohkamsing S, van Gurp RJ, Oosterhuis JW, van der Saag PT, Looijenga LH. Lack of Bcllo mutations in testicular germ cell tumours and derived cell lines. Br I Cancer 1999; $80: 1571-4$.

15. van Leeuwen FW, Burbach JP, Hol EM. Mutations in RNA: a first example of molecular misreading in Alzheimer's disease. Trends Neurosci 1998; $21: 331-5$.

16. Benne R. RNA editing: how a message is changed. Curr Opin Genet Dev 1996; 6: 221-31. 17. Gronbaek K, Straten PT, Ralfkiaer E, et al. Somatic Fas mutations in non-Hodgkin's lymphoma: association with extranodal disease and autoimmunity. Blood 1998; 92: 3018-24.

\section{Gilles Salles}

Jeune équipe universitaire, Pathologie des cellules lymphoïdes, Université ClaudeBernard Lyon-1, 69495 Pierre-Bénite Cedex, France.

\section{$40^{e}$ journée annuelle de nutrition et de diététique CNIT - PARIS LA DÉFENSE}

(amphithéâtre Léonard-de-Vinci)

\section{Vendredi 28 janvier 2000}

\section{Président : Professeur Bernard GUY-GRAND}

Vice-Présidents : Professeur Arnaud BASDEVANT,

Professeur Bernard MESSING, Professeur Gérard SLAMA

Secrétaire Générale : Marie-France CARRIÉ-MOYAL

Fondateurs : Professeur Henri BOUR, Professeur Maurice DEROT, Docteur Guy HERAUD

Membre d'honneur : Docteur Michel RATHERY

Accueil des participants à partir de 8 h 30

\section{La dénutrition en I'an 2000}

Présidents modérateurs : Professeur Bernard MESSING - Professeur Luc CYNOBER

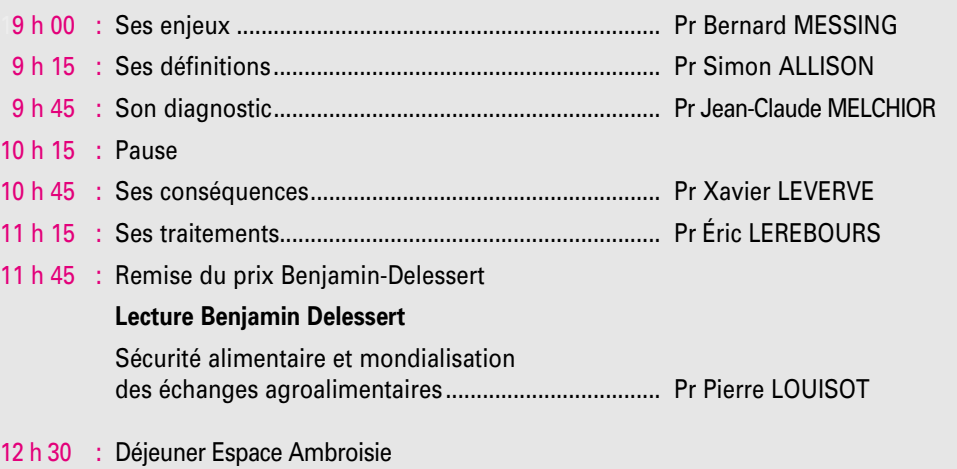

\section{Les apports nutritionnels conseillés en l'an 2000}

Présidents modérateurs : Professeur Bernard GUY-GRAND - Professeur Arnaud BASDEVANT

$14 \mathrm{~h} 30$ : Pour quoi ? Pour qui ? Pr Joël MENARD

$15 \mathrm{~h} 00$ : Comment les déterminer? Mr Philippe PATUREAU MIRAND

$15 \mathrm{~h} 30$ : Que nous enseigne le modèle de la vitamine $C$ ?...... Dr Inès BIRLOUEZ-ARAGON

$16 \mathrm{~h} 00$ : Comment les mettre en place? Pr Ambroise MARTIN

$16 \mathrm{~h} 30$ : Fin de séance

\section{Renseignements}

Pour tous renseignements s'adresser au

Secrétariat de la Journée Annuelle de Nutrition et de Diététique 30, rue de Lübeck, 75116 Paris

Tél. : 33145534169 de 10 h à 18 h 